\title{
Comparison of endostatin combined with PT-DC versus bevacizumab combined with PT-DC in the first-line treatment of advanced lung adenocarcinoma: a retrospective propensity score- matched cohort study
}

\author{
Xiaoling Chen, Jun Nie, Ling Dai, Weiheng Hu, Jie Zhang, Jindi Han, Xiangjuan Ma, Guangming Tian, \\ Sen Han, Di Wu, Yang Wang, Jieran Long, Ziran Zhang, Jian Fang \\ Department of Thoracic Oncology II, Key Laboratory of Carcinogenesis and Translational Research (Ministry of Education/Beijing), Peking \\ University Cancer Hospital and Institute, Beijing, China \\ Contributions: (I) Conception and design: X Chen, J Fang; (II) Administrative support: J Fang; (III) Provision of study materials or patients: All \\ authors; (IV) Collection and assembly of data: X Chen, W Hu; (V) Data analysis and interpretation: X Chen, W Hu, J Fang; (VI) Manuscript writing: \\ All authors; (VII) Final approval of manuscript: All authors. \\ Correspondence to: Jian Fang. Department of Thoracic Oncology II, Key Laboratory of Carcinogenesis and Translational Research (Ministry of \\ Education/Beijing), Peking University Cancer Hospital and Institute, Beijing, China. Email: fangiian5555@yeah.net.
}

Background Endostatin and bevacizumab have been approved for the first-line treatment of advanced
non-small-cell lung cancer (NSCLC) patients in China; however, the clinical outcomes for each drug
combined with platinum-based doublet chemotherapy (PT-DC) have not yet been directly compared. This
study sought to assess the clinical outcomes of the 2 drugs combined with PT-DC in the first-line treatment
of patients with advanced lung adenocarcinoma.

Methods: This retrospective cohort study examined the clinical data of patients with metastatic or recurrent lung adenocarcinoma (LUAD) treated with endostatin or bevacizumab combined with PT-DC as the first-line treatment from October 2010 to November 2019. Propensity score matching (PSM) was performed using a 1:1 ratio nearest neighbor algorithm. The effectiveness and safety outcomes for the 2 groups were evaluated.

Results: A total of 202 patients were enrolled in the study. Of these, the endostatin group comprised 124 patients and the bevacizumab group comprised 78 patients; 67 pairs of patients were identified after PSM. The progression-free survival (PFS) and overall survival (OS) of patients treated with PT-DC + endostatin and PT-DC + bevacizumab were compared [(PFS: before PSM 4.8 vs. 6.5 months, P=0.741; after PSM 6.5 vs. 6.1 months, $\mathrm{P}=0.402$ ), (OS: before PSM 21.1 vs. 39.3 months, $\mathrm{P}=0.912$; after PSM 23.6 vs. 39.3 months, $\mathrm{P}=0.579)$ ]. The objective response rates (ORRs) and disease control rates (DCRs) of the 2 groups were comparable (37.7\% vs. 50.7\%, $\mathrm{P}=0.094 ; 89.6 \%$ vs. $92.5 \%, \mathrm{P}=0.545)$. Adverse events (AEs) $\geq$ grade 3 were not observed in the PT-DC + endostatin group. Three $(3.8 \%)$ cases of AEs $\geq$ grade 3 were observed the PT-DC + bevacizumab group, comprising hypertension $(n=1)$, proteinuria $(n=1)$, hemoptysis $(n=1)$.

Conclusions: This retrospective analysis showed that in first-line treatments, PT-DC + endostatin and PT-DC + bevacizumab appear to produce similar anti-tumor activities in patients with metastatic or recurrent lung adenocarcinoma. PT-DC + bevacizumab tended to result in worse adverse reactions than PT$\mathrm{DC}+$ endostatin.

Keywords: Anti-angiogenic agents; bevacizumab; endostatin; advanced lung adenocarcinoma; propensity score matching (PSM)

Submitted May 10, 2021. Accepted for publication Jun 29, 2021.

doi: 10.21037/apm-21-1401

View this article at: https://dx.doi.org/10.21037/apm-21-1401 


\section{Introduction}

Lung cancer is the leading cause of cancer-related deaths worldwide (1-3). It has an incidence of 31.5 per 100,000 men and 14.6 per 100,000 women (3). In 2018, approximately 774,000 new cases of lung cancer were reported, and approximately 690,000 people died of lung cancer in China (3). Non-small-cell lung cancer (NSCLC) accounts for about $80 \%$ of all lung cancers; $75 \%$ of NSCLC patients are in an advanced stage at diagnosis (4).

Platinum-based doublet chemotherapy (PT-DC) is the standard regimen used to treat advanced NSCLC, but the corresponding 5 -year survival rate is still very low $(<5 \%)(5)$. In recent decades, more and more evidence has shown that neovascularization plays a key role in the growth, proliferation, and metastasis of a variety of solid tumors (6-10). Anti-angiogenic drugs can simultaneously act on tumor microenvironments to degenerate the existing tumor vasculature and inhibit the formation of new blood vessels $(9,11)$. Clinical data has shown that a combination of antiangiogenic drugs and other systemic therapeutic drugs (including chemotherapy) can improve the anti-tumor effect, delay drug resistance, and any adverse reactions can be managed (12). Anti-angiogenic drugs are indispensable in the treatment of cancers.

Bevacizumab is a humanized monoclonal antibody with high affinity to VEGF. The VEGF family includes VEGF-A, VEGF-B, VEGF-C, VEGF-D and placental growth factor (13). The specific antiangiogenic mechanism of endostatin has not been fully elucidated at present. The main mechanism of the antitumor effect is that endostatin acts on the VEGF receptor KDR/Flk-1 and inhibits the signal transduction of VEGF (14). In addition, endostatin seems to play a multitarget antiangiogenic role by regulating expression of vascular endothelial growth factor and activity of proteolytic enzymes, indirectly leading to the quiescence or reduction of tumors.

Endostation is an anti-angiogenic inhibitor independently developed by China, and it is approved by China's State Food and Drug Administration (CFDA) for NSCLC. Bevacizumab is approved by FDA and CFDA for NSCLC, colorectal cancer, renal cancer and so on. At present, 2 anti-angiogenic drugs (bevacizumab and endostatin) have been approved for the first-line treatment of advanced NSCLC patients by CFDA. However, to date, no comparisons appear to have been made of the 2 antivascular drugs combined with PT-DC in the first-line treatment of advanced lung adenocarcinoma.

Previous studies have evaluated extended use of endostatin combined with PT-DC and evaluated bevacizumab combined with PT-DC with or without bevacizumab maintenance $(15,16)$. The results both suggested that extended use of anti-angiogenic therapy after anti-angiogenic drug combined with PT-DC improved the effectiveness (PFS and/or OS). But the clinical outcomes of endostatin combined with PT-DC and bevacizumab combined with PT-DC have not yet been directly compared. in particular, the comparison of the effectiveness of extended use of two anti-angiogenic drugs after they combined with PT-DC. This study sought to assess the clinical outcomes of the 2 drugs combined with PT-DC (especially the clinical outcomes of extended use of anti-angiogenic drugs after they combined with PT-DC) in the first-line treatment of patients with advanced lung adenocarcinoma.

In this study, we retrospectively analyzed the effectiveness and safety of the 2 anti-angiogenic drugs combined with PT-DC in the first-line treatment of advanced lung adenocarcinoma. To our knowledge, this appears to be the first study to directly compare the effectiveness of endostatin or bevacizumab combined with PT-DC in the first-line treatment of patients with advanced lung adenocarcinoma. We present the following article in accordance with the STROBE reporting checklist (available at https://dx.doi.org/10.21037/apm-21-1401).

\section{Methods}

\section{Study design and patient enrollment}

This retrospective, single-center study used the clinical data of Chinese patients with advanced adenocarcinoma who received first-line PT-DC with endostatin or bevacizumab from October 2010 to November 2019 at the Second Department of Thoracic Medicine of Beijing Cancer Hospital (HIS). All procedures performed in this study involving human participants were in accordance with the Declaration of Helsinki (as revised in 2013) (17). Individual consent for this retrospective analysis was waived. The study was approved by the Ethics Committee of Peking University Cancer Hospital and Institute (No.: 2019KT43).

To be enrolled in this study, patients had to meet the following inclusion criteria: (I) have lung adenocarcinoma (with cytological or histological confirmation) and have been treated with endostatin or bevacizumab (endostatin $15 \mathrm{mg}$, i.v., on days $1-14$ and bevacizumab $7.5 \mathrm{mg} / \mathrm{kg}$, i.v., on day 1 ; treatment was administered at 3 -week intervals); 


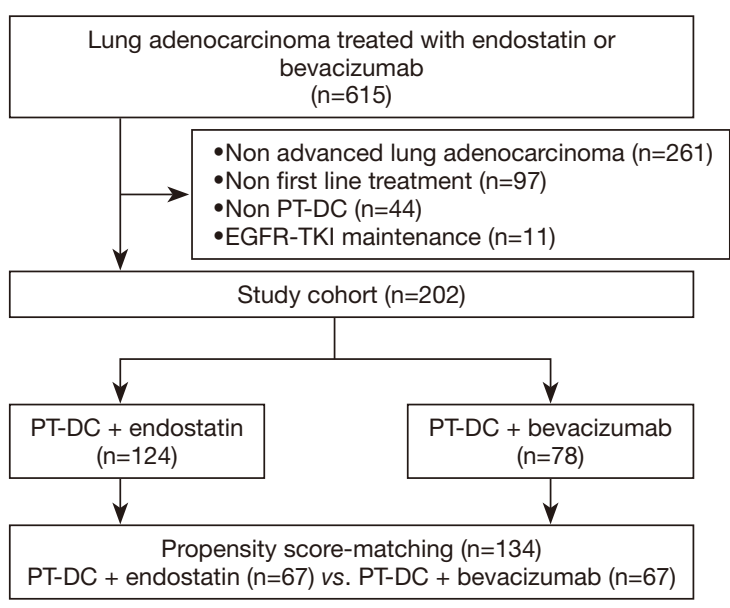

Figure 1 Flow chart for the selection of patients. PT-DC, platinum-based doublet chemotherapy.

(II) have undergone a radiographic examination confirming advanced lung adenocarcinoma (metastatic or recurrent); and (III) have received first-line treatment of PT-DC.

The chemotherapy regimens in this study included pemetrexed plus cisplatin/carboplatin, gemcitabine plus cisplatin/carboplatin, paclitaxel plus cisplatin/carboplatin, and docetaxel plus cisplatin/carboplatin. Chemotherapy was administered at 3-week intervals: pemetrexed $\left(500 \mathrm{mg} / \mathrm{m}^{2}\right.$, i.v., on day 1$)$, gemcitabine $\left(1,250 \mathrm{mg} / \mathrm{m}^{2}\right.$, i.v., on days 1 and 8$)$, paclitaxel $\left(175 \mathrm{mg} / \mathrm{m}^{2}\right.$, i.v., on day 1$)$, and platinum [cisplatin, $75 \mathrm{mg} / \mathrm{m}^{2}$ over 2 days, i.v., on days $1-2$ or carboplatin (area under the curve, 4-5), i.v., on day 1]. The choice of regimens was mainly based on the time of treatment, renal function, and economic factors. Patients in the pemetrexed plus cisplatin/carboplatin+endostatin group received endostatin plus pemetrexed or endostatin maintenance treatment after induction chemotherapy. Patients in the pemetrexed plus cisplatin/carboplatin+bevacizumab group received bevacizumab plus pemetrexed or bevacizumab maintenance treatment after induction chemotherapy. Patients with endostatin or bevacizumab combined with other chemotherapy regimens received endostatin or bevacizumab maintenance therapy after induction chemotherapy. Treatment continued until the disease progressed, withdrawal, or intolerable toxicity.

Patients were excluded from the study if they met the following exclusion criteria: (I) had received second-line therapy or above with endostatin or bevacizumab after the administration of the first-line treatment; (II) had received another combination therapy; (III) had received maintenance treatment with epidermal growth factor receptor (EGFR) tyrosine kinase inhibitors. The flowchart for patient enrollment in this study is presented in Figure 1.

\section{Data collection}

Patient data (chief complaint, disease history, physical examination, imaging examinations, and biochemical laboratory tests) were collected retrospectively through the information system of HIS, which has a comprehensive electronic patient chart system that is fully indexed and searchable (18). The number of cases during the study period determined the sample size.

According to the Response Evaluation Criteria in Solid Tumors version 1.1 (19), the response of each patient was classified as a complete response (CR), a partial response $(\mathrm{PR})$, stable disease $(\mathrm{SD})$, or $\mathrm{PD}$. The objective response rate $(\mathrm{ORR})$ was $(\mathrm{CR}+\mathrm{PR}) /(\mathrm{CR}+\mathrm{PR}+\mathrm{SD}+\mathrm{PD}) \times 100 \%$. The disease control rate (DCR) was $(\mathrm{CR}+\mathrm{PR}+\mathrm{SD}) /$ $(\mathrm{CR}+\mathrm{PR}+\mathrm{SD}+\mathrm{PD}) \times 100 \%$. Progression-free survival $(\mathrm{PFS})$ was defined as the time from the start of treatment to progression or death from any cause. Overall survival (OS) was defined as the time from the start of treatment to death from any cause. After completing the treatment, the patients attended follow-up clinical visits and telephone interviews to ascertain progression or survival every 3 months. The values of the patients whose follow-up data were missing and those who did not progress were treated as censored values. The censored time was the last follow-up appoint at which it was confirmed that a patient had not progressed or died. The follow-up period ceased on July 27, 2020.

Toxicities were evaluated according to the National Cancer Institute-Common Toxicity Criteria for Adverse Events version 4.03 (20).

\section{Statistical analysis}

Propensity score (PS) methods estimate the treatment effect by modelling the relationship between confounders and treatment assignment, then offer certain advantages over more traditional regression methods to control for confounding by indication in observational studies. The PS is the probability for a subject to receive a treatment conditional on a set of baseline characteristics (confounders) and it is commonly estimated using logistic regression, and it is used to match patients with similar distribution of confounders so that difference in outcomes gives unbiased estimate of treatment effect. The propensity score matching (PSM) is not limited by the number of events, and this 
method may be warranted when the number of confounders is large, or the number of outcomes is small (21).

The propensity score matching (PSM) method was applied to reduce any selection bias that might arise due to the large number of covariates among patients who received endostatin or bevacizumab in this study. Each variable was multiplied by a coefficient that was calculated using a logistic regression analysis, and the sum of these values was considered to be the propensity score for individual patients. PSM was performed using the nearest matching method with a 1:1 matching ratio. The caliper was set to 0.2 to balance the potential selective bias.

In the present study, the variables included in the PSM were sex, age, smoking history, Eastern Cooperative Oncology Group (ECOG) score, driver gene variants [EGFR/anaplastic lymphoma kinase (ALK)], brain metastasis, liver metastasis, chemotherapy regimens, and cycles of anti-vascular therapy.

The categorical data are presented as numbers (percentages) and were analyzed using the chi-square test or Fisher's exact test. Kaplan-Meier curves and the logrank test were used to compare the survival data between the groups. A univariable and multivariable Cox regression model was used for the analysis of PFS and OS. The data filtering method used in the multivariable Cox regression was a backward stepwise regression (likelihood ratio). $\mathrm{P}$ values $<0.05$ were considered statistically significant. The research goals mentioned above were achieved by conducting analyses using IBM SPSS Statistics (Version 23.0; IBM, Armonk, NY, USA).

\section{Results}

\section{Clinical characteristics}

A search identified 615 Chinese patients with lung adenocarcinoma who had been treated with endostatin or bevacizumab at the hospital between October 2010 and November 2019. Of these, 202 eligible patients were enrolled according to their treatment regimens. The PTDC + endostatin group comprised 124 patients, and the PT$\mathrm{DC}+$ bevacizumab group comprised 78 patients. The most commonly used chemotherapy regimens were pemetrexed plus cisplatin/carboplatin $(105 ; 52.0 \%)$, gemcitabine plus cisplatin/carboplatin $(72 ; 35.6 \%)$, paclitaxel plus cisplatin/carboplatin $(25 ; 12.4 \%)$. The median number of chemotherapy cycles was 4 .

The median follow-up period was 15.9 (range,
1.4-93.7) months. Several imbalances in characteristics existed between the PT-DC + endostatin and PT-DC + bevacizumab patients before PSM, including imbalances in the chemotherapy regimens $(\mathrm{P}<0.001)$, EGFR status $(\mathrm{P}<0.001)$, ALK status $(\mathrm{P}=0.002)$, and cycles of anti-vascular therapy $(\mathrm{P}<0.001)$. Patients in $\mathrm{PT}-\mathrm{DC}+$ bevacizumab group had more pemetrexed plus cisplatin/carboplatin treatment, less EGFR positive, less ALK positive and longer anti-vascular treatment time than those in the PT-DC + endostatin group. There were no significant differences in the distribution of the other baseline characteristics between the 2 groups. After PSM, 67 pairs of patients were 1:1 matched in the 2 groups, and all baseline characteristics were well balanced. Table 1 sets out details of each confounding variable before and after matching.

\section{Effectiveness analyses}

\section{Survival profile}

During the follow-up period, 164 progressions [107 of $124(86.3 \%)$ in the PT-DC + endostatin group, and 57 of $78(73.1 \%)$ in the PT-DC + bevacizumab group], and 119 deaths (90 of $124(72.6 \%)$ in the PT-DC + endostatin group, and 29 of 78 in $(37.2 \%)$ the PT-DC + bevacizumab group) were reported.

The results of the univariable and multivariable Cox proportional hazards regression analyses for the entire cohort are summarized in Table 2. In the multivariable analyses, smoking history and cycles of anti-vascular therapy were independent prognostic factors for PFS and OS. Patients who had no smoking history and had undergone $\geq 4$ cycles of anti-vascular drug therapy had a better prognosis. No EGFR mutation was an independent favorable predictor for PFS, and an ECOG score of 0-1 was an independent favorable predictor for OS. Therapy combined with bevacizumab was a favorable predictor for PFS in the univariable $(\mathrm{P}=0.013)$ but not the multivariable analysis $(\mathrm{P}=0.747)$. Neither endostatin nor bevacizumab was a predictor of OS in the univariable or multivariable analyses.

Before PSM, a difference in PFS was observed between patients treated with PT-DC + endostatin and those treated with PT-DC + bevacizumab [PFS: 4.8 vs. 6.5 months, hazard ratio (HR) $0.67,95 \%$ confidence interval (CI), 0.48 0.92, $\mathrm{P}=0.013$; see Figure 2A]. Before PSM, no significant difference in OS was observed between patients treated with PT-DC + endostatin and those treated with PT-DC + bevacizumab (OS: 21.1 vs. 39.3 months, HR 0.75, 95\% CI, 
Table 1 Clinicopathological characteristics of patients before and after PSM

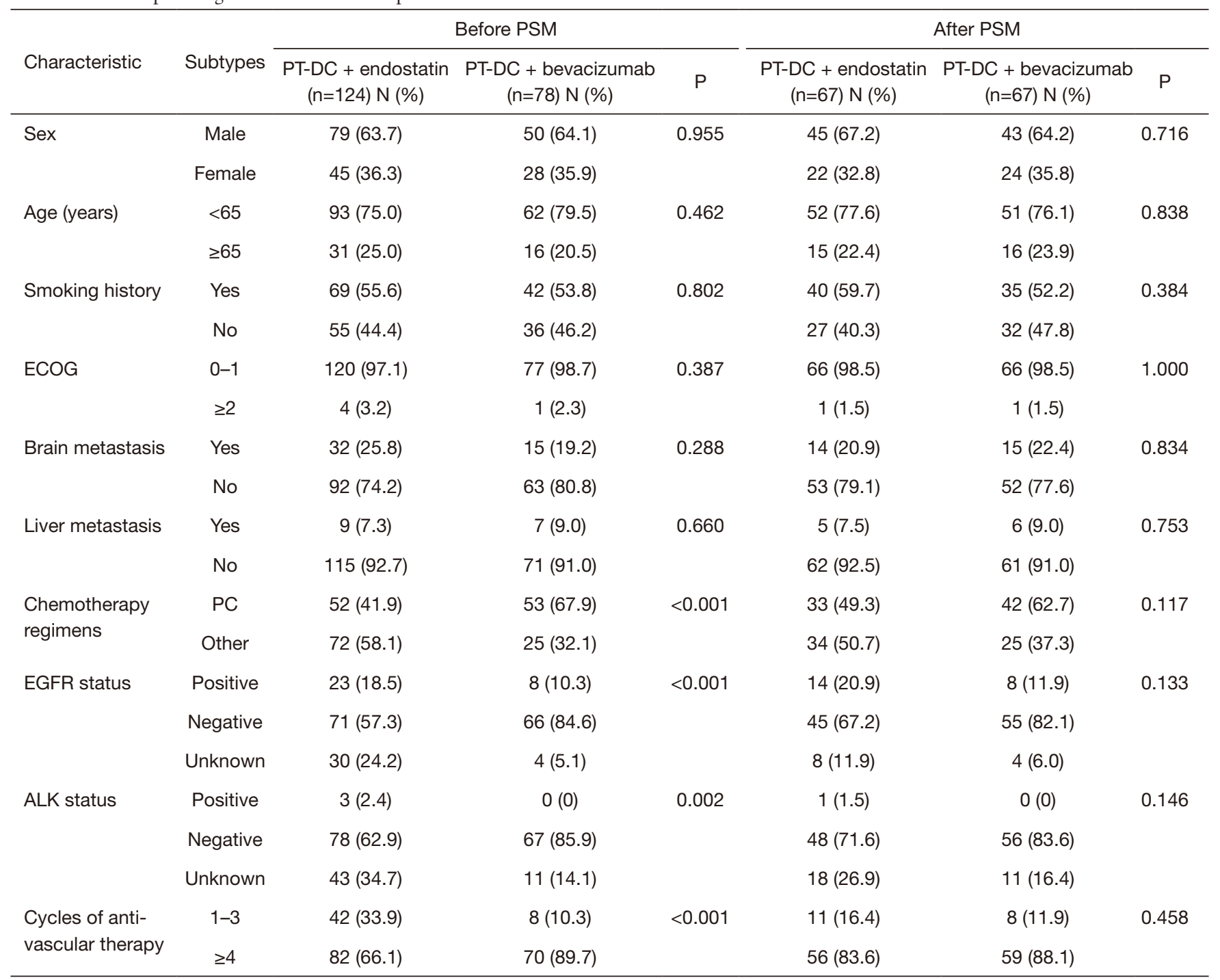

PSM, propensity score matching; PT-DC, platinum-based doublet chemotherapy; ECOG, Eastern Cooperative Oncology Group; EGFR, epidermal growth factor receptor; ALK, anaplastic lymphoma kinase.

\subsection{9-1.15, $\mathrm{P}=0.186$; see Figure 2B).}

PSM was performed to balance confounding covariates across treatment groups. After PSM, bevacizumab combined with PT-DC was not associated with improved survival outcomes (PFS: 6.5 vs. 6.1 months, HR 0.85, 95\% CI, $0.58-1.25, \mathrm{P}=0.402$; OS: 23.6 vs. 39.3 months, HR 0.87, 95\% CI, 0.53-1.43, P=0.579; see Figure $3 A, B)$.

\section{Response profile}

As Table 3 shows, after PSM, 67 pairs of patients were identified, and patients' responses were evaluated. The CR, $\mathrm{PR}, \mathrm{SD}$, and PD rates of the PT-DC + endostatin group and PT-DC + bevacizumab group were $0 \%, 37.3 \%, 52.2 \%$, and $10.4 \%$, and $0 \%, 50.7 \%, 41.8 \%$, and $7.5 \%$, respectively $(\mathrm{P}=0.289)$. Additionally, the ORRs and DCRs of the PT$\mathrm{DC}+$ endostatin group were compared with those of the PT-DC + bevacizumab group $(37.7 \%$ vs. $50.7 \%, \mathrm{P}=0.094$; $89.6 \%$ vs. $92.5 \%, \mathrm{P}=0.545)$.

\section{Safety analyses}

The prevalence of serious adverse events (AEs) associated with anti-vascular therapy, including hypertension, proteinuria, arterial and venous thromboembolic events, 
Table 2 Univariable and multivariable analyses of PFS and OS

\begin{tabular}{|c|c|c|c|c|c|c|}
\hline Variable & \multicolumn{3}{|c|}{ Univariate analysis } & \multicolumn{3}{|c|}{ Multivariate analysis } \\
\hline \multicolumn{7}{|l|}{ PFS } \\
\hline Sex & 1.09 & $0.79-1.49$ & 0.613 & 0.94 & $0.55-1.64$ & 0.837 \\
\hline Age (years) & 1.06 & $0.74-1.53$ & 0.751 & 1.21 & $0.83-1.76$ & 0.318 \\
\hline ECOG & 1.70 & $0.69-4.15$ & 0.247 & 2.11 & $0.85-5.25$ & 0.110 \\
\hline Brain metastasis & 1.30 & $0.90-1.87$ & 0.165 & 1.16 & $0.79-1.72$ & 0.457 \\
\hline Liver metastasis & 1.43 & $0.83-2.48$ & 0.202 & 1.63 & $0.94-2.85$ & 0.084 \\
\hline Chemotherapy regimens & 1.24 & $0.91-1.70$ & 0.166 & 1.05 & $0.75-1.47$ & 0.774 \\
\hline Cycles of anti-vascular therapy & 0.12 & $0.08-0.17$ & $<0.001$ & 0.11 & $0.07-0.16$ & $<0.001$ \\
\hline Anti-vascular drug & 0.67 & $0.48-0.92$ & 0.013 & 1.06 & $0.74-1.53$ & 0.741 \\
\hline \multicolumn{7}{|l|}{ os } \\
\hline Sex & 1.90 & $1.28-2.82$ & 0.001 & 1.38 & $0.80-2.36$ & 0.246 \\
\hline Age (years) & 1.07 & $0.70-1.64$ & 0.764 & 1.15 & $0.74-1.79$ & 0.522 \\
\hline Smoking history & 2.08 & $1.43-3.01$ & $<0.001$ & 2.39 & $1.62-3.52$ & $<0.001$ \\
\hline $\mathrm{ECOG}$ & 3.76 & $1.36-10.36$ & 0.011 & 3.41 & $1.23-9.47$ & 0.019 \\
\hline Brain metastasis & 1.26 & $0.84-1.90$ & 0.266 & 1.07 & $0.69-1.67$ & 0.768 \\
\hline Anti-vascular drug & 0.75 & $0.49-1.15$ & 0.186 & 0.91 & $0.59-1.42$ & 0.912 \\
\hline
\end{tabular}

ECOG, Eastern Cooperative Oncology Group; EGFR, epidermal growth factor receptor; ALK, anaplastic lymphoma kinase; PFS, progression-free survival; OS, overall survival.

gastrointestinal perforations, hemorrhage (e.g., hemoptysis and bleeding within the central nervous system), and arrhythmia, were investigated. These adverse reactions are of special concern to anti-angiogenic drugs (endostation and bevacizumab). In the PT-DC + endostatin group, there were 0 cases of hypertension, 0 cases of proteinuria, 4 (3.2\%) cases of arterial and venous thromboembolic events, 2 (1.6\%) cases of hemorrhage, 4 (3.2\%) cases of arrhythmia, and $0 \mathrm{AEs} \geq$ grade 3 . In the PT-DC + bevacizumab group, there were $2(2.6 \%)$ cases of hypertension, $5(6.4 \%)$ cases of proteinuria, 5 cases $(6.4 \%)$ of arterial and venous thromboembolic events, $3(3.8 \%)$ case of hemorrhage, 1 (1.3\%) case of arrhythmia, and $3(3.8 \%)$ AEs $\geq$ grade 3 , comprising 1 case of hypertension, 1 case of proteinuria, and 1 case of hemoptysis.

\section{Discussion}

Anti-angiogenic therapy (of endostatin or bevacizumab) combined with PT-DC is considered a first-line treatment 

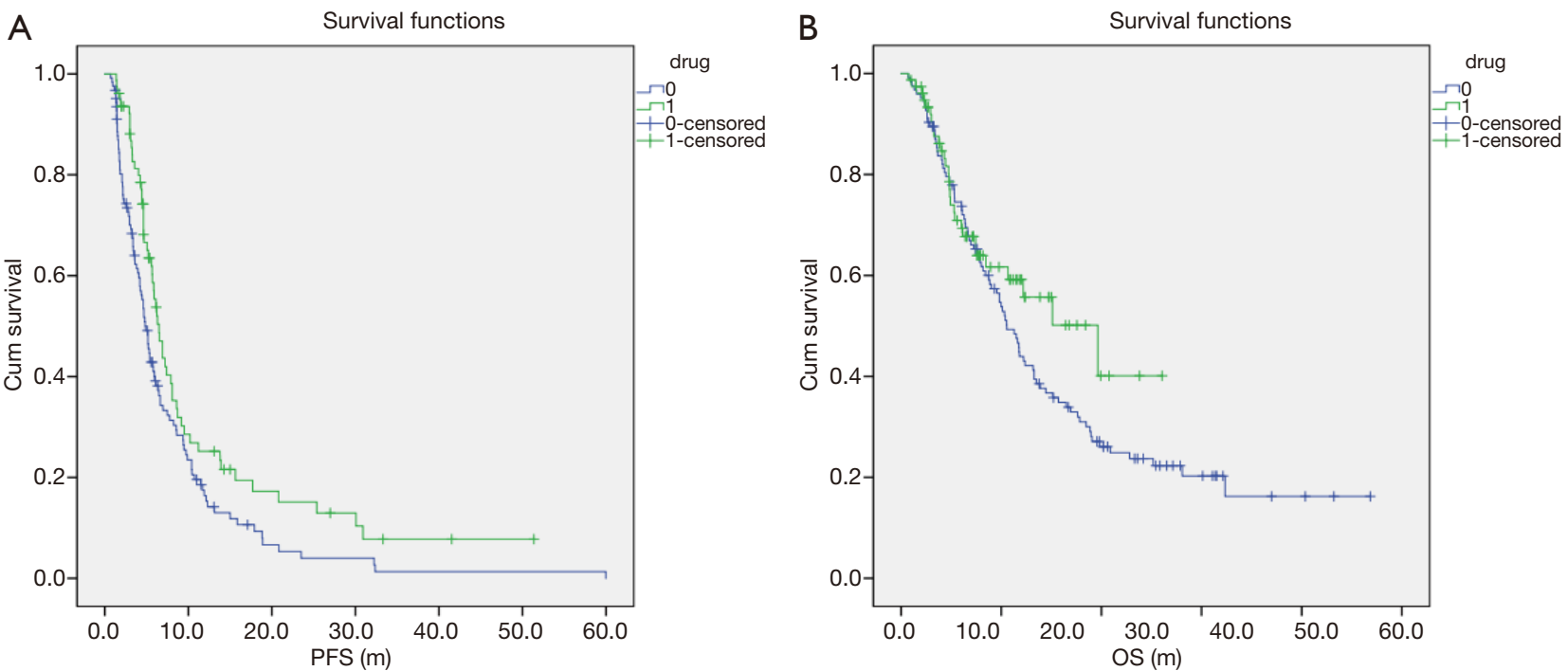

Figure 2 Kaplan-Meier survival curves of PFS and OS between patients by treatment with PT-DC + endostatin versus PT-DC + bevacizumab before PSM. (A) PFS: 4.8 months, 4.1-5.6 months vs. 6.5 months, 5.5-7.5 months (P=0.013). (B) OS: 21.1 months, 17.6-24.6 months vs. 39.3 months, $18.5-60.0$ months $(\mathrm{P}=0.186)$. PFS, progression-free survival; OS, overall survival; PSM, propensity score matching.
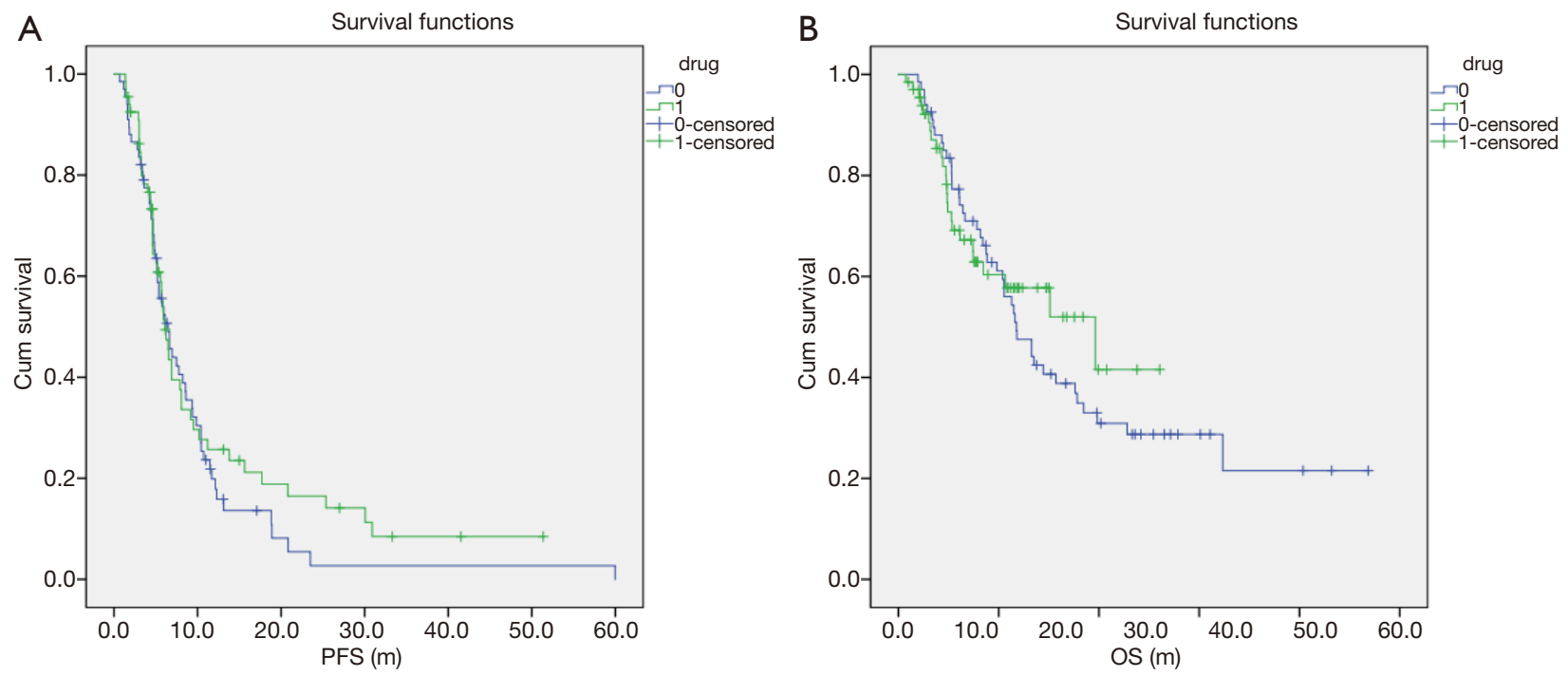

Figure 3 Kaplan-Meier survival curves of PFS and OS between patients by treatment with PT-DC + endostatin versus PT-DC + bevacizumab after PSM. (A) PFS: 6.5 months, 4.8-8.2 months vs. 6.1 months, 5.2-7.1 months (P=0.402). (B) OS: 23.6 months, 17.8-29.3 months vs. 39.3 months 14.4-64.1 months $(\mathrm{P}=0.579)$. PFS, progression-free survival; OS, overall survival; PSM, propensity score matching.

for advanced NSCLC in China. However, only limited clinical trials have been conducted comparing endostar combined with PT-DC and bevacizumab combined with PT-DC, which has limited the clinical application of antiangiogenic agents combined with chemotherapy in the treatment of NSCLC. In this matched-pair case-control study, we demonstrated that PT-DC + endostatin and PT-DC + bevacizumab appear to have similar outcomes in patients with advanced NSCLC who received firstline treatments. The ORRs and DCRs of the PT-DC + endostatin group were comparable to those of the PT-DC + bevacizumab group $(37.7 \%$ vs. $50.7 \%, \mathrm{P}=0.094 ; 89.6 \%$ vs. 
Table 3 Effectiveness between PT-DC + endostatin group and PT$\mathrm{DC}+$ bevacizumab group after PSM

\begin{tabular}{lccc}
\hline Effectiveness & $\begin{array}{c}\text { PT-DC }+ \\
\text { endostatin }(\mathrm{n}=67) \\
\mathrm{N}(\%)\end{array}$ & $\begin{array}{c}\text { PT-DC }+ \\
\text { bevacizumab }(\mathrm{n}=67)\end{array}$ & $\mathrm{P}$ \\
\hline $\mathrm{NR}$ & $0(0)$ & 0.289 \\
$\mathrm{PR}$ & $25(37.3)$ & $34(50.7)$ & \\
SD & $35(52.2)$ & $28(41.8)$ & \\
PD & $7(10.4)$ & $5(7.5)$ & \\
ORR & $25(37.3)$ & $34(50.7)$ & 0.094 \\
DCR & $60(89.6)$ & $62(92.5)$ & 0.545 \\
\hline
\end{tabular}

PSM, propensity score matching; PT-DC, platinum-based doublet chemotherapy; CR, complete response; PR, partial response; SD, stable disease; PD, progressive disease; ORR, objective response rate; $\mathrm{DCR}$, disease control rate.

92.5\%, $\mathrm{P}=0.545)$. The survival outcomes of patients treated with PT-DC + endostatin or PT-DC + bevacizumab were also comparable (PFS: 6.5 vs. 6.1 months, HR 0.85, 95\% CI, $0.58-1.25, \mathrm{P}=0.402$; OS: 23.6 vs. 39.3 months, HR $0.87,95 \%$ CI, 0.53-1.43, $\mathrm{P}=0.579)$. Before PSM, PT-DC + bevacizumab was a favorable predictor of $\mathrm{PFS}$ in the univariable $(\mathrm{P}=0.013)$ but not the multivariable analysis $(\mathrm{P}=0.747)$. Neither endostatin nor bevacizumab was a predictor of OS in the univariable or in multivariable analyses.

Endostatin (endostar), a C-terminal fragment of collagen $\mathrm{XVIII}$, is a broad-spectrum angiogenesis inhibitor that can interfere with the pro-angiogenic action of growth factors, such as VEGFs and fibroblast growth factors (FGFs), resulting in the inhibition of endothelial proliferation, angiogenesis, and tumor growth $(6,22)$. It was approved by China's SFDA for the treatment of NSCLC in 2005 . The decision was mainly based on a phase-III randomized, double-blind, multicenter trial (23) that compared first-line treatments with vinorelbine plus cisplatin/carboplatin (NP) plus endostar or NP alone in advanced NSCLC patients. The ORRs in the NP plus endostar group $(\mathrm{N}=322)$ and the $\mathrm{NP}$ alone group $(\mathrm{N}=164)$ were $35.4 \%$ and $19.5 \%(\mathrm{P}<0.01)$, respectively, the median time to progression was 6.3 and 3.6 months $(\mathrm{P}<0.001)$, respectively, and the median survival time was 14.8 and 9.9 months $(\mathrm{P}<0.001)$, respectively.

Bevacizumab (Avastin) is a recombinant, humanized monoclonal immunoglobulin $\mathrm{G}$ antibody. It has a high affinity in binding with all VEGF-A isoforms circulating in the blood, and neutralizes VEGF's biologic activity via the steric blocking of its binding with vascular endothelial growth factor receptor (VEGFR) (24,25). Based on the results of 2 phase-III trials, it has been approved for metastatic non-squamous-NSCLC in combination with PT-DC. In a multicenter E4599 trial, patients in the bevacizumab group had both longer OS (12.3 vs. 10.3 months; HR: $0.79 ; \mathrm{P}=0.003)$ and PFS (6.2 vs. 4.5 months; HR: 0.66; $\mathrm{P}<0.001)$ and higher ORRs $(35 \%$ vs. $15 \% ; \mathrm{P}<0.001)$ than those in the control group (26). A second phase-III AVAiL trial showed that compared to a combination of a placebo and chemotherapy, a combination of bevacizumab and chemotherapy conferred a significant benefit in terms of PFS, at both high (6.5 vs. 6.1 months; HR: 0.82; $\mathrm{P}=0.03)$ and low (6.7 vs. 6.1 months; HR: 0.75; $\mathrm{P}=0.003)$ doses. However, this benefit did not result in a significant improvement in OS, which only increased from 13.1 months in the placebo group to 13.6 and 13.4 months in the low- and high-dose bevacizumab groups, respectively $(\mathrm{P}=0.42$ for the low-dose bevacizumab group; $\mathrm{P}=0.76$ for the high-dose bevacizumab bevacizumab) (27). A metaanalysis of 2,194 patients from phase-II and phase-III trials, confirmed that combining bevacizumab with platinumbased chemotherapy in the first-line treatment of advanced NSCLC provided significant benefits to patients in terms of both OS and PFS, compared to those provided by chemotherapy alone. The estimated HRs for OS and PFS were 0.90 (95\% CI: 0.81-0.99; $\mathrm{P}=0.03)$ and 0.72 (95\% CI: $0.66-0.79 ; \mathrm{P}<0.001)$, respectively (28).

In the present study, we mainly analyzed the effectiveness of antiangiogenic drugs (endostatin $v s$. bevacizumab) combined with chemotherapy by PSM method. The ORR and DCR of the PT-DC + endostatin group were comparable to those of the PT-DC + bevacizumab group. PFS and OS in the PT-DC + endostatin group and the PT$\mathrm{DC}+$ bevacizumab group were comparable. In relation to the ORRs and OS values, PT-DC + bevacizumab was better than PT-DC + endostatin. There may be a number of reasons for these results. First, the use of pemetrexed plus cisplatin/carboplatin was more common in the PT-DC + bevacizumab group than the PT-DC + endostatin group. Second, the number of cycles of anti-vascular drugs in the PT-DC + bevacizumab group was greater than that in the PT-DC + endostatin group. Only one previous network meta-analysis compared the effectiveness of endostatin and bevacizumab combined with chemotherapy, and the results suggested that bevacizumab combined with chemotherapy regimens has better therapeutic effect on ORR while endostatin combined with chemotherapy may have better effects on OS and PFS for the treatment of NSCLC 
patients (29). The possible reason for difference between studies may be that meta-analysis included nonrandomized controlled trials, and the quality of the trails included in the analysis was not uniform. In the present study, the PSM method used to control and eliminate the selective bias. In conclusion, the effectiveness of PT-DC + endostatin was at least as good as that of PT-DC + bevacizumab.

Additionally, hypertension, proteinuria, arterial and venous thromboembolic events, hemorrhage, and arrhythmia were more common in the PT-DC + bevacizumab group, while arrhythmia was more common in the PT-DC + endostatin group. AEs $\geq$ grade 3 were only found in the PT$\mathrm{DC}+$ bevacizumab group. The adverse reactions related to the anti-angiogenic therapy of PT-DC + endostatin tended to be superior to those of PT-DC + bevacizumab.

This study had the following limitations: it was retrospective in nature, had a small sample size, and was undertaken at a single institution. These factors affected the statistical power of our data and could have resulted in biases. PSM was used to match pairs to minimize the potential selection bias; however, it also affected the sample size. Additionally, the predictive value of the balanced variables in this study need to be further explored in prospective settings.

To our knowledge, this study appears to be the first study to directly compare the effectiveness of endostatin or bevacizumab combined with PT-DC in the first-line treatment of patients with advanced lung adenocarcinoma. This study provided essential clinical insights into a realworld Chinese cohort with advanced lung adenocarcinoma. In relation to first-line treatments, PT-DC + endostatin and PT-DC + bevacizumab appear to have similar antitumor activities in terms of response rates, PFS, and OS in patients with metastatic or recurrent NSCLC. PT-DC + bevacizumab tended to result in worse adverse reactions than PT-DC + endostatin.

\section{Acknowledgments}

Funding: Funding for this study was received from Special Funds for Clinical Research of Wu Jieping Medical Foundation, grant number: 320.6750.19030.

\section{Footnote}

Reporting Checklist: The authors have completed the STROBE reporting checklist. Available at https://dx.doi. org/10.21037/apm-21-1401
Data Sharing Statement: Available at https://dx.doi. org/10.21037/apm-21-1401

Conflicts of Interest: All authors have completed the ICMJE uniform disclosure form (available at https://dx.doi. org/10.21037/apm-21-1401). The authors have no conflicts of interest to declare.

Ethical Statement: The authors are accountable for all aspects of the work in ensuring that questions related to the accuracy or integrity of any part of the work are appropriately investigated and resolved. All procedures performed in this study involving human participants were in accordance with the Declaration of Helsinki (as revised in 2013). Individual consent for this retrospective analysis was waived. The study was approved by the Ethics Committee of Peking University Cancer Hospital and Institute (No.: 2019KT43).

Open Access Statement: This is an Open Access article distributed in accordance with the Creative Commons Attribution-NonCommercial-NoDerivs 4.0 International License (CC BY-NC-ND 4.0), which permits the noncommercial replication and distribution of the article with the strict proviso that no changes or edits are made and the original work is properly cited (including links to both the formal publication through the relevant DOI and the license). See: https://creativecommons.org/licenses/by-nc-nd/4.0/.

\section{References}

1. Siegel RL, Miller KD, Jemal A. Cancer statistics, 2016. CA Cancer J Clin 2016;66:7-30.

2. Arenberg D. Update on screening for lung cancer. Transl Lung Cancer Res 2019;8:S77-87.

3. Bray F, Ferlay J, Soerjomataram I, et al. Global cancer statistics 2018: GLOBOCAN estimates of incidence and mortality worldwide for 36 cancers in 185 countries. CA Cancer J Clin 2018;68:394-424.

4. Rivera MP, Mehta AC, Wahidi MM. Establishing the diagnosis of lung cancer: Diagnosis and management of lung cancer, 3rd ed: American College of Chest Physicians evidence-based clinical practice guidelines. Chest 2013;143:e142S-e165S.

5. Pallis AG, Georgoulias V. Is there a standard regimen for first-line treatment of advanced/metastatic Non-SmallCell Lung Cancer? What has meta-analyses contributed to today's standard of care. Lung Cancer 2012;75:269-74. 
6. Folkman J. Antiangiogenesis in cancer therapy-endostatin and its mechanisms of action. Exp Cell Res 2006;312:594-607.

7. Hanahan D, Weinberg RA. Hallmarks of cancer: the next generation. Cell 2011;144:646-74.

8. Carmeliet P, Jain RK. Molecular mechanisms and clinical applications of angiogenesis. Nature 2011;473:298-307.

9. Viallard C, Larrivee B. Tumor angiogenesis and vascular normalization: alternative therapeutic targets. Angiogenesis 2017;20:409-26.

10. Nowak-Sliwinska P, Alitalo K, Allen E, et al. Consensus guidelines for the use and interpretation of angiogenesis assays. Angiogenesis 2018;21:425-532.

11. Jain RK. Normalization of Tumor Vasculature: An Emerging Concept in Antiangiogenic Therapy. Science 2005;307:58-62.

12. Tian $W$, Cao C, Shu L, et al. Anti-Angiogenic Therapy in the Treatment of Non-Small Cell Lung Cancer. Onco Targets Ther 2020;13:12113-29.

13. Chikaishi Y, Tanaka F, Honda Y, et al. Prospects for the future of epidermal growth factor receptor-tyrosine kinase inhibitors in combination with bevacizumab. Transl Cancer Res 2020;9:1307-10.

14. Kim YM, Hwang S, Kim YM, et al. Endostatin blocks vascular endothelial growth factor-mediated signaling via direct interaction with KDR/Flk-1. J Biol Chem 2002;277:27872-9.

15. Hu W, Fang J, Nie J, et al. Efficacy and safety of extended use of platinum-based doublet chemotherapy plus endostatin in patients with advanced nonsmall cell lung cancer. Medicine 2016;95:e4183.

16. Nørøxe DS, Wallerek S, Sørensen JB. Platinum-based doublet chemotherapy plus bevacizumab without bevacizumab maintenance in advanced non-small cell lung cancer (NSCLC). Anticancer Res 2013;33:3275-8.

17. World Medical Association Declaration of Helsinki Ethical Principles for Medical Research Involving Human Subjects. Available online: https://www.wma.net/wpcontent/uploads/2016/11/DoH-Oct2013-JAMA.pdf

18. Ding R, Zhu D, He P, et al. Comorbidity in lung cancer patients and its association with medical service cost and treatment choice in China. BMC Cancer 2020;20:250.

19. Eisenhauer EA, Therasse P, Bogaerts J, et al. New response evaluation criteria in solid tumours: revised RECIST guideline (version 1.1). Eur J Cancer 2009;45:228-47.

20. U.S. Department of Health and Human Services. National Institutes of Health NCI. Common Terminology Criteria for Adverse Events (CTCAE). Version 4.0. Published:
May 28, 2009 (v4. 03: June 14, 2010). Available online: https://evsncinihgov/ftp1/CTCAE/CTCAE_403/ CTCAE_403_2010-06-14_QuickReference_85x11pdf. Accessed June 28, 2020(2010).

21. Benedetto U, Head SJ, Angelini GD, et al. Statistical primer: propensity score matching and its alternatives. Eur J Cardiothorac Surg 2018;53:1112-7.

22. O'Reilly MS, Boehm T, Shing Y, et al. Endostatin: An Endogenous Inhibitor of Angiogenesis and Tumor Growth. Cell 1997;88:277-85.

23. Wang J, Sun Y, Liu Y, et al. Results of randomized, multicenter, double-blind phase III trial of rh-endostatin (YH-16) in treatment of advanced non-small cell lung cancer patients. Zhongguo Fei Ai Za Zhi 2005;8:283-90.

24. Willett CG, Boucher Y, di Tomaso E, et al. Direct evidence that the VEGF-specific antibody bevacizumab has antivascular effects in human rectal cancer. Nat Med 2004;10:145-7.

25. Presta LG, Chen H, O'Connor SJ, et al. Humanization of an Anti-Vascular Endothelial Growth Factor Monoclonal Antibody for the Therapy of Solid Tumors and Other Disorders. Cancer Res 1997;57:4593-9.

26. Sandler A, Gray R, Perry MC, et al. Paclitaxel-Carboplatin Alone or with Bevacizumab for Non-Small-Cell Lung Cancer. N Engl J Med 2006;355:2542-50.

27. Reck M, von Pawel J, Zatloukal P, et al. Overall survival with cisplatin-gemcitabine and bevacizumab or placebo as first-line therapy for nonsquamous non-small-cell lung cancer: results from a randomised phase III trial (AVAiL). Ann Oncol 2010;21:1804-9.

28. Soria JC, Mauguen A, Reck M, et al. Systematic review and meta-analysis of randomised, phase II/III trials adding bevacizumab to platinum-based chemotherapy as first-line treatment in patients with advanced non-small-cell lung cancer. Ann Oncol 2013;24:20-30.

29. Li Y, Yi Y, Lin A, et al. A comparison of the efficacy of antiangiogenic agents combined with chemotherapy for the treatment of non-small cell lung cancer: a network meta-analysis. Cancer Cell Int 2020;20:548.

Cite this article as: Chen $\mathrm{X}$, Nie J, Dai L, Hu W, Zhang J, Han J, Ma X, Tian G, Han S, Wu D, Wang Y, Long J, Zhang Z, Fang J. Comparison of endostatin combined with PTDC versus bevacizumab combined with PT-DC in the first-line treatment of advanced lung adenocarcinoma: a retrospective propensity score-matched cohort study. Ann Palliat Med 2021;10(7):7847-7856. doi: 10.21037/apm-21-1401 